\title{
Classification of Laser Interferometer Seismic Signal Data based on K-means Clustering
}

\author{
Dongsun Choi, Eunhwan Oh, Wooram Lee, and Kwanho You
}

\begin{abstract}
The mainly used devices to detect earthquakes are velocimeters and accelerometers. However, it is necessary to detect seismic waves accurately in micro-seismic wave measurement. A heterodyne laser interferometer is a high resolution detection device. Therefore, the laser interferometer is used as a seismometer. In micro-seismic signal analysis, it is required to discriminate earthquakes from explosions since micro-seismic waves have small magnitudes. To classify seismic waves, the body-wave amplitude $\left(A_{p}\right)$ and the surface-wave amplitude $\left(A_{s}\right)$ data are used. K-means clustering is implemented and then earthquake and explosion events are discriminated by using these data.
\end{abstract}

Keywords - Body-wave amplitude, K-means clustering, Laser Interferometer, Seismic signal, Surface-wave amplitude

\section{Introduction}

Many attempts on understanding for nature have been persisted constantly. Earthquake is one of the major research areas. Analyses of earthquakes have been extended to various parts such as distance of epicenter, magnitude measurement, frequency and intensity analysis. As an earthquake early warning, a micro-seismic wave has become a notable subject recently.

Usually, the mainly used devices to measure seismic wave are velocimeters and accelerometers. In the micro-seismic signal detection, however, the demands for more precision measurement apparatus increases a lot. High accuracy of measurement leads to decrease in errors and to increase in reliability. A heterodyne laser interferometer is an ultraprecision measurement device with superiority such as long

\section{Dongsun Choi}

Sungkyunkwan University

Korea

Eunhwan Oh

Sungkyunkwan University

Korea

\section{Wooram Lee}

Sungkyunkwan University

Korea

\section{Kwanho You}

Sungkyunkwan University

Korea measurement range, adjustable set up, and contactless measurement. Therefore, seismic signal can be detected exactly with a noticeable error reduction.

Since micro-seismic waves have small magnitudes, it is necessary to discriminate a detected seismic wave from explosions. There have been many efforts on methods to classify between earthquakes and explosions. Dahy [1] suggested a complexity method and a spectral P-wave amplitude method which discriminates between explosion and earthquake. Kebeasy [2] proposed criterion as a method of discriminating between natural earthquakes and nuclear explosions. Ford [3] explained aftershock characteristics as methods of discriminating explosions from earthquakes. Bian [4] implemented a research on the support vector machine classification of earthquake and explosion.

In this paper, we propose that a K-means clustering discrimination algorithm between the earthquakes and the explosions based on the heterodyne laser interferometer. The heterodyne laser interferometer detects a seismic wave more accurately than seismometers such as velocimeters and accelerometers. With $A_{p}$ and $A_{s}$, the measured seismic wave discriminates the earthquakes from the explosions by the Kmeans clustering. The result of the clustering guarantees that a new collected seismic wave is determined whether it is the earthquakes or not.

This paper is organized as follows. In section 2, a measurement of seismic waves with the heterodyne laser interferometer is introduced. Section 3 explains the K-means clustering as a classification method between earthquakes and explosions. In section 4, the effectiveness of the proposed algorithm is proved through some simulations. Finally, conclusions are presented in section 5 .

\section{Heterodyne Laser Interferometer}

In this section, the laser interferometer system is introduced to detect earthquakes. Many advantages exist, such as high resolution and wide range of measurement in the laser interferometer. Using those strengths, it is possible to measure seismic wave precisely. Figure 1 presents a heterodyne laser interferometer system.

In the heterodyne laser interferometer, laser head emits a beam which consists of two orthogonal frequency components. The laser beam is divided into two signals which are reference and measurement signal while it passes through a beam splitter (BS). Both signals are indicated by $A \omega_{1}$ and $B \omega_{2}$. 


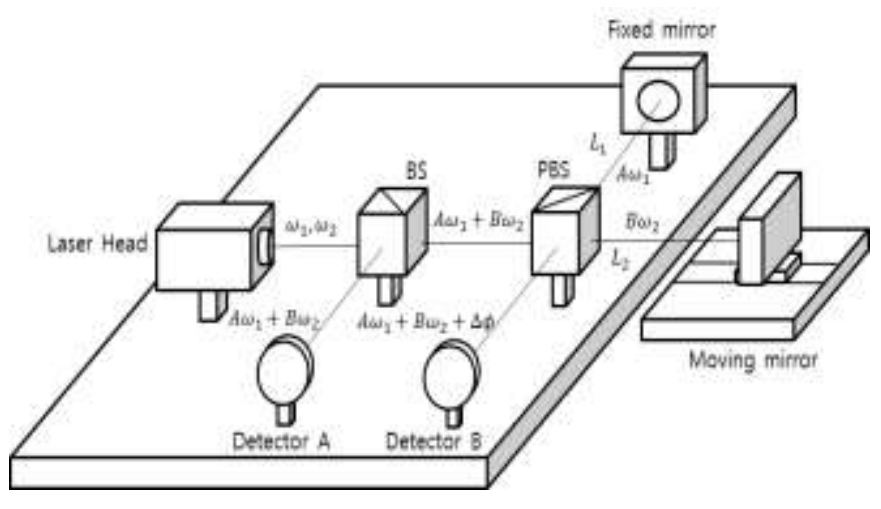

Figure 1. Seismic signal detection using a heterodyne laser interferometer.

The reference signal is collected by detector $A$. The electric fields of a reference signal are expressed as follows,

$$
\begin{aligned}
& E_{A_{1}}=\frac{1}{\sqrt{2}} A \exp \left[i\left(\omega_{1} t+\phi_{A}\right)\right] \\
& E_{A_{2}}=\frac{1}{\sqrt{2}} B \exp \left[i\left(\omega_{2} t+\phi_{B}\right)\right]
\end{aligned}
$$

where $A, B$ are amplitudes, $\omega_{1}, \omega_{2}$ are frequencies, and $\phi_{A}$, $\phi_{B}$ are initial phases of two beams, respectively. Intensity of a reference signal obtained by the electric field is

$$
\begin{aligned}
I_{r} & \propto\left(E_{A_{1}}+E_{A_{2}}\right)\left(E_{A_{1}}+E_{A_{2}}\right)^{*} \\
& =\frac{1}{2}\left(A^{2}+B^{2}\right)+A B\left[\cos \left(\Delta \omega t+\left(\phi_{B}-\phi_{A}\right)\right)\right]
\end{aligned}
$$

where $\Delta \omega$ means $\Delta \omega=\omega_{2}-\omega_{1}$.

The measurement signal which passes through a polarization beam splitter (PBS) is divided into two signals. One is reflected by a fixed mirror, and the other is reflected by a moving mirror along paths, $L_{1}$ and $L_{2}$, respectively. Reflected signals pass again through the PBS and are recombined. The reunified signal is collected by detector $B$. The electric fields of the measurement signal are represented as follows,

$$
\begin{aligned}
& E_{B_{1}}=\frac{1}{\sqrt{2}} A \exp \left[i\left(\omega_{1} t+\phi_{A}\right)\right] \\
& E_{B_{2}}=\frac{1}{\sqrt{2}} B \exp \left[i\left(\omega_{2} t+\phi_{B}+\Delta \phi\right)\right]
\end{aligned}
$$

where $\Delta \phi$ is a phase difference occurred by Doppler effect in moving mirror. Intensity of measurement signal which is acquired by the electric field is

$$
\begin{aligned}
I_{m} & \propto\left(E_{B_{1}}+E_{B_{2}}\right)\left(E_{B_{1}}+E_{B_{2}}\right)^{*} \\
& =\frac{1}{2}\left(A^{2}+B^{2}\right)+A B\left[\cos \left(\Delta \omega t+\left(\phi_{B}-\phi_{A}\right)+\Delta \phi\right)\right]
\end{aligned}
$$

To concentrate on the phase difference $\Delta \phi$ between $I_{r}$ and $I_{m}$, DC parts of reference and measurement signal are deleted by using a high-pass filter

$$
\begin{aligned}
& I_{r, a c} \propto A B \cos (\Delta \omega t) \\
& I_{m, a c} \propto A B \cos (\Delta \omega t+\Delta \phi)
\end{aligned}
$$

In order to obtain the phase value, lock-in amplifier is used. Procedures of lock-in amplifier are as following steps. The first step is to generate two intensity signals by signal processing of a multiplication of $I_{r, a c}$ and $I_{m, a c}$, and a multiplication of $I_{r, a c}$ shifted by 90 degree and $I_{m, a c}$. In the second step, AC parts of the signals are deleted with a lowpass filter. The results are as follows,

$$
\begin{aligned}
& I_{x} \propto \frac{A B}{2} \cos (\Delta \phi) \\
& I_{y} \propto \frac{A B}{2} \sin (\Delta \phi)
\end{aligned}
$$

The phase difference $\Delta \phi$ can be extracted by a trigonometrical function.

$$
\Delta \phi=\tan ^{-1}\left(\frac{I_{y}}{I_{x}}\right)
$$

Using the phase difference, the distance difference $\Delta L$ between fixed mirror and moving mirror is acquired.

$$
\Delta L=\frac{\Delta \phi \lambda}{4 \pi n}
$$

where $n$ is the refractive index, and $\lambda$ is an average wavelength.

\section{K-means clustering}

In this section, a K-means clustering is proposed as an algorithm to classify seismic events into earthquakes and explosions. The K-means clustering is an effective method to assemble some groups which are comprised of elements with similar properties. Respective event data are assigned to a specific cluster which makes the minimum Euclidean distance among centroids. The clustering proceeds quickly under conditions that the number of clusters is preselected and the centroids are set randomly [5].

With K-means clustering, the seismic waves can be discriminated as earthquakes or explosions. There are various considerable conditions for the discriminating. Among the options, a body-wave amplitude $\left(A_{p}\right)$ and a surface-wave amplitude $\left(A_{s}\right)$ are used to classify into earthquakes and explosions. The body-wave amplitude and the surface-wave amplitude are also called as a p-wave amplitude and a s-wave 
amplitude, respectively. By using the two amplitudes as parameters, K-means clustering can be proceeded.

The clustering process works to minimize the total Euclidean distance between the cluster centroids and the elements which represents as follows

$$
J=\sum_{j=1}^{k} \sum_{i=1}^{N}\left|v_{i}-c_{j}\right|^{2}
$$

where $v_{i}$ is $i$-th data where $i=1,2, \ldots, N$, and $c_{j}$ is the $j-t h$ order of a centroid. A procedure for K-means clustering is as the following steps. First, the number of clusters $(k)$ is selected and the initial locations of $k$ centroids are set randomly. Secondly, the distances from a specific datum to all centroids are compared. After the comparison, the datum belongs to the closest cluster.

This process is executed for all data. Third, new centroids are determined as follows

$$
c_{j}=\frac{1}{k_{j}} \sum_{i \in c_{j}} v_{i}
$$

The several processes are repeated until the total Euclidean distance $(J)$ is minimized. Figure 2 represents a flow chart of the clustering.

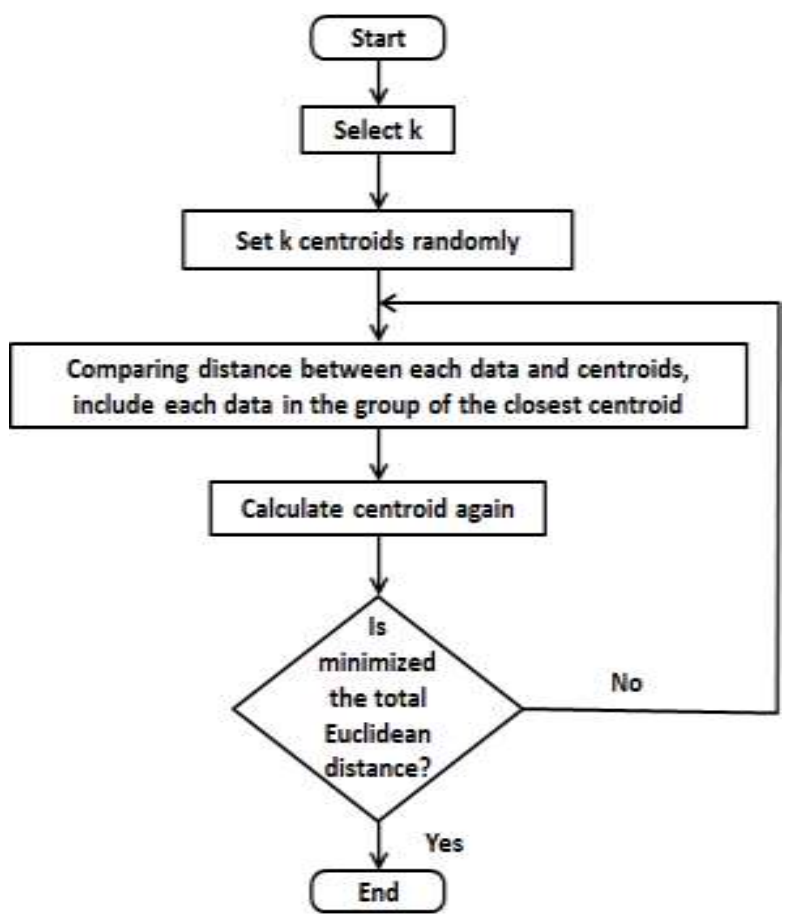

Figure 2. Clustering flow chart

With pre-collected seismic signal data, two clusters can be prepared which represent earthquakes and explosions. Then a new seismic event can be discriminated by comparing the distance from two centroids.

\section{Simulation}

In this section, the effectiveness of the K-means clustering is demonstrated with some simulation results. The seismic signal is detected using the laser interferometer. The laser head used in experiments is WT307b from Wave-Tronics with $632.991 \mathrm{~nm}$ wavelength $(\lambda)$. The refractive index is $n=1.00000002665$ and the amplitudes of $A$ and $B$ are set to1 volt. As a translation stage to generate seismic waves, the linear transducer (ST-PSA6520) is used.

The K-means clustering discriminates earthquakes from explosion by using two parameters which are the body-wave amplitude $\left(A_{p}\right)$ and the surface-wave amplitude $\left(A_{s}\right)$. The body-wave amplitude is the peak value of p-wave and the surface-wave amplitude is the peak value of s-wave.

In simulation, the $\mathrm{K}$-means clustering is implemented to discriminate earthquakes from explosions. The data set which includes 16 earthquake events and 16 explosion events is generated by the linear transducer and measured by a laser interferometer. Figures 3 and 4 show samples of seismic waves.

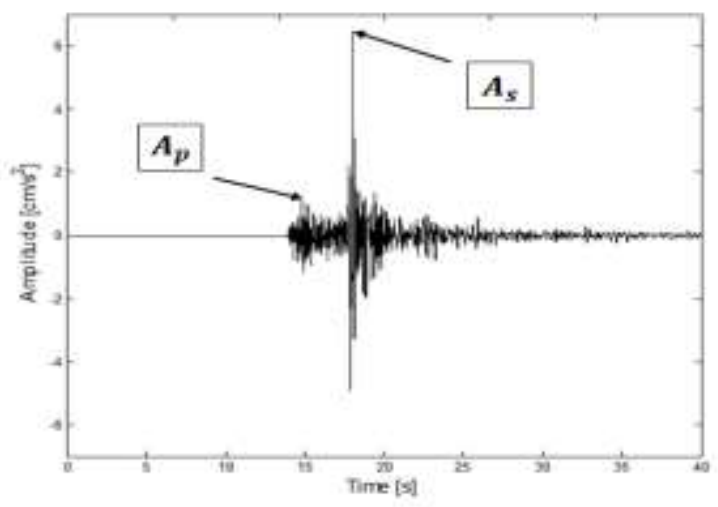

Figure 3. Earthquake wave

Figure 3 presents a sample of the earthquake wave which is measured using a laser interferometer. The body-wave amplitude is the value of $1.135 \mathrm{~cm} / \mathrm{s}^{2}$ at about 15 second and the surface-wave amplitude is the value of $6.489 \mathrm{~cm} / \mathrm{s}^{2}$ at about 18 second.

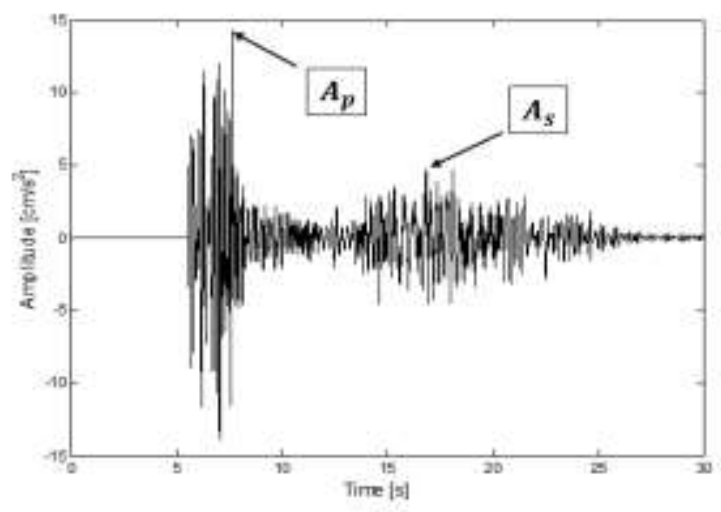

Figure 4. Explosion wave 
A seismic wave sample in figure 4 is the explosion wave. Body-wave amplitude is the value of $14.24 \mathrm{~cm} / \mathrm{s}^{2}$ at about 8 second and surface-wave amplitude is the value of $5.229 \mathrm{~cm} / \mathrm{s}^{2}$ at about 17 second.

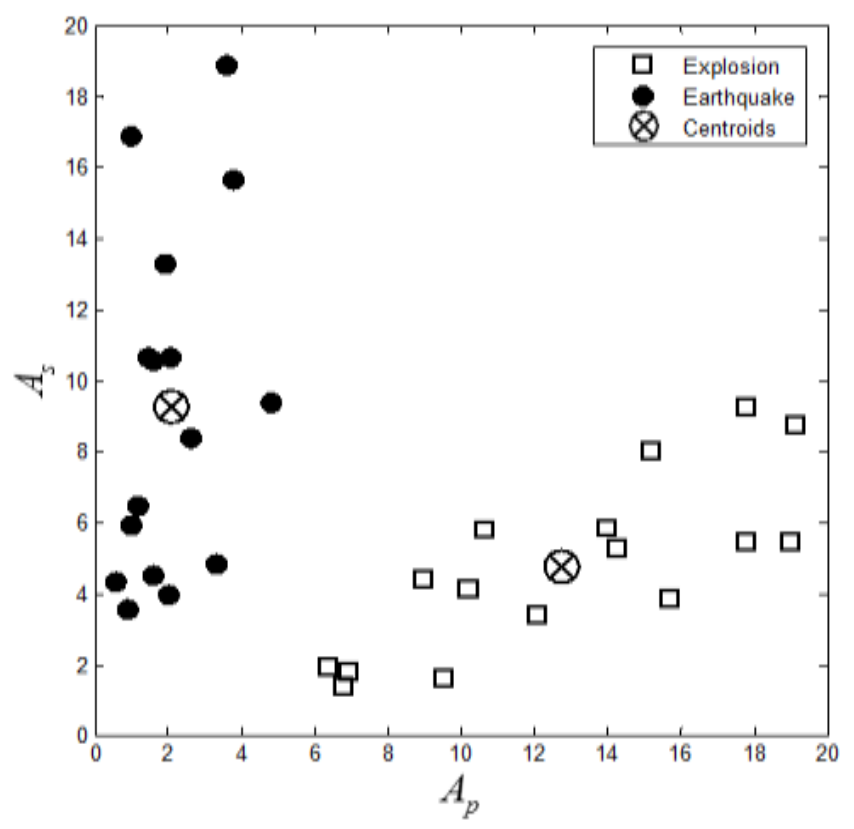

Figure 5. Clustering earthquakes and explosions

The number of clusters $(k)$ is two. The $x$-axis is a bodywave amplitude and the $y$-axis is a surface-wave amplitude. Clustering completes as two groups which are explosions and earthquakes. In figure 5 , the circle marks represent the earthquakes and the square marks are the explosions. The centroids of the earthquakes and explosions are displayed as the circle with a cross mark. The earthquake events are located on the top-left space and the explosion events are located on the bottom-right space. Using a body-wave amplitude and a surface-wave amplitude, the seismic events are classified effectively.

\section{v. Conclusion}

This paper suggested a seismic signal classification method based on the K-means clustering. The laser interferometer was used as a seismometer to detect a seismic wave precisely. Two parameters which are a body-wave amplitude and a surface-wave amplitude are used to classify the seismic wave measured by the laser interferometer. The K-means clustering discriminated earthquakes and explosions clearly by using the parameters. As a new seismic event occurs, the event is determined whether it is the earthquakes or not by calculating the distance between the event and the centroids.

\section{Acknowledgement}

This research was supported by Basic Science Research Program through the National Research Foundation of Korea (NRF) funded by the Ministry Education, Science and Technology (2013R1A1A2006728).

\section{References}

[1] A. S. Dahy and H. G. Hassib, "Discriminating nuclear explosions from earthquakes at teleseismic distances," European Journal of Applied Sciences, vol. 1, pp. 47-52, 2009.

[2] R. M. Kebeasy, A. I. Hussein, and S. A. Dahy, "Discrimination between natural earthquakes and nuclear explosions using the Aswan Seismic Network," ANNALI DI GEOFISICA, vol. 41, no. 2, pp. 127-140, June 1998.

[3] S. R. Ford and W. R. Walter, "Aftershock characteristics as a means of discriminating explosions from earthquakes," Bulletin of the Seismological Society of America, vol. 100, no. 1, pp. 364-376, May 2009.

[4] Y. Bian, H. Huang, and T. Wang, "A research on the SVM classification of earthquake and explosion based upon seismic wave features," International Journal of Digital Content Technology \& its Applications, vol. 8, pp. 53-60, Dec. 2014.

[5] I. A. Yusoff and N. A. M. Isa, "Two-dimensional clustering algorithms for image segmentation," WSEAS Transactions on Computers, vol. 10, pp. 332-342, Oct. 2011. 